\title{
Recovery of Leptospires After Dry Storage For Ten Years
}

\author{
D. I. ANNEAR \\ Department of Microbiology, Royal Perth Hospital, Perth, Western Australia
}

\begin{abstract}
Nine strains of leptospires representing six serotypes were dried in vacuo from the liquid state and stored at $4 \mathrm{C}$. Viable organisms were recovered from all dried cultures during and after 10 years of storage. Motile forms were not observed immediately after rehydration, but they became apparent after several days of incubation.
\end{abstract}

In their recent paper on the preservation of leptospires by freezing, Alexander et al. (1) reviewed the literature on this topic and also commented that attempts to preserve these organisms by freeze-drying have rarely been successful. Although this may be true, it is difficult to gauge the extent of this failure since there has been little documentation of it. On the other hand, successful recoveries of leptospires after drying and storage have been claimed by Annear $(2,3,6)$ and by Otsuka and Monako (8).

In one of these reports (3), it was recorded that dried cultures were prepared by drying liquid leptospiral suspensions in vacuo on the walls of small glass tubes and that viable organisms were recovered from these preparations after storage for 5 years (6). Subsequent tests showed the organisms to be viable after several additional years of storage at $4 \mathrm{C}$, but these experiments have now terminated since no further tubes remain for testing. However, a second series of experiments was done 10 years ago with a modified drying method and on a wider range of leptospires. The method was derived from previous work on the preservation of other microorganisms $(4,5)$. Briefly, it involved drying liquid leptospiral suspensions in vacuo on quartz fibers contained in small glass tubes. This paper describes the preparation of these dried cultures and the results from tests on their viability during and at the end of the 10-year storage period.

\section{MATERIALS AND METHODS}

Organisms. Nine strains of leptospires of six different serotypes were dried. The serotypes were icterohaemorrhagiae (two strains), canicola (two strains), pomona (two strains), tarassovi, australis, and zanoni. The organisms were grown in Korthof medium (7) at $30 \mathrm{C}$ and harvested for drying 2 to 3 days after the initial turbidity was observed.
Suspensions for drying. Suspensions were prepared from both uncentrifuged cultures and from centrifuged deposits of $20-\mathrm{ml}$ culture volumes. Centrifuging was done for $30 \mathrm{~min}$ at $5,000 \times \mathrm{g}$. In the former experiments, $50 \%$ (wt/vol) glucose was added to the culture to yield a final carbohydrate concentration of $5 \%(\mathrm{wt} / \mathrm{vol})$; in the latter experiments, the centrifuged deposits were mixed with equal volumes of $10 \%$ (wt/vol) glucose.

Drying and storage. Small glass tubes with tufts of quartz wool in the bottoms and cotton-wool plugs in the necks were prepared and sterilized by autoclaving at $15 \mathrm{lb} / \mathrm{in}^{2}$ for $20 \mathrm{~min}(4,5)$. Two-drop volumes of the suspensions were inoculated on the quartz fibers, and drying was then carried out in vacuo on a manifold over $\mathrm{P}_{2} \mathrm{O}_{5}$ and at a pressure of approximately $10^{-2}$ torr. The tubes were exposed externally to ambient atmosphere, and no attempt was made to control the drying temperature (4). The tubes were sealed in vacuo after $24 \mathrm{~h}$ on the manifold and were stored at $4 \mathrm{C}$.

Recovery. The dried cultures were rehydrated with and cultured in about $3 \mathrm{ml}$ of Korthof medium contained in small, screw-capped plastic tubes. The tubes were incubated at $30 \mathrm{C}$ and inspected daily for turbidity.

\section{RESULTS AND DISCUSSION}

At least two experiments were carried out for each organism, one with an uncentrifuged culture and one with a centrifuged deposit. Dried cultures were examined randomly during the 10-year storage period and, at the end of this time, at least three tubes of each experiment were tested. Except for an occasional culture spoiled by contamination, all of the tubes yielded positive cultures. The dried cultures prepared from the centrifuged deposits yielded turbid cultures in 4 to 7 days, whereas with the unconcentrated suspensions the range was extended by 1 to 2 days. In some control experiments, it was shown that both inocula 
rinsed from the fibers before drying and resuspended in Korthof medium yielded turbid growth in 2 to 3 days. There was, therefore, an appreciable retardation of growth after drying.

Upon microscope examination immediately after rehydration, no motile organisms could be detected, but after 1 to 3 days of incubation such forms were invariably seen, and turbid cultures could then be predicted with confidence. The motile organisms, viewed by darkfield microscopy, presented the typical leptospire appearance and were indistinguishable in this respect from the organisms in the undried cultures. Macroscopically the recovery cultures gave the light, silky turbidity characteristic of members of the genus. There are no data concerning the fraction of organisms that survive and yield viable reproductive forms. Likewise, it is unknown whether the culture is derived from undetected, and therefore of small numbers, forms that are actively motile immediately after rehydration or from inactive forms that regain their motility during incubation.

Quartz fibers were selected as drying surfaces in this work because of their relative inertness and because of the large area they provide (4). However, in addition to the experiments recorded here, others were done with the same range of organisms but with small glass beads instead of quartz wool, and similar results were obtained. These results, together with those obtained by using the walls of the glass ampules themselves (3), show that several surfaces are suitable for the purpose, although minor effects may be attributable to them. Moreover, it would appear from the results in this laboratory, where drying was done from the liquid state, and from those of Otsuka and Monako (8), who dried their cultures from the frozen state, that the drying temperatures for successful preservation are not critical. Direct comparisons would obviously be necessary for reliable information on this factor.

Drying from the liquid state has two distinct advantages over freeze-drying: it is simpler and it can be done with firm cotton-wool plugs in the necks of the tubes, a procedure that reduces the risks of infecting the worker and contaminating the dried culture. In freeze-drying methods, such plugging is not feasible because of the restricted vapor flow that tends to thaw the frozen suspension during dehydration. On the other hand, an advantage of freeze-drying lies in the increased volume of suspension that can be dried, a factor that may be important when survival is low.

In the absence of motile forms in the early phases of rehydration, there would be an understandable reluctance to regard the drying as successful; such observations may account for an apparent failure with drying techniques in other laboratories. However, the fact that such appearances do not necessarily indicate unsuccessful drying has been clearly shown in the present studies, where consistent cultural recoveries from dried cultures have been obtained.

Although no critical comparisons have been made, there appeared to be little, if any, increase in the recovery periods as storage progressed, and it seems most likely that the dried cultures will contain viable organisms for a considerable time to come.

The relative recovery periods obtained with centrifuged and uncentrifuged suspensions would appear to be inversely related to cell concentrations, and the advantage of the shorter recovery period would seem to justify the optional step of centrifuging the cultures.

Early experiments in this work indicated that storage of dried cultures at room temperature yielded unreliable recoveries after several months, and no further comparisons have been made with storage at $4 \mathrm{C}$. Also, although several other suspending media, including glutamate, have been tested, none appeared to give as consistently reliable results as glucose. It must be remembered that the components of Korthof medium, including rabbit serum, are also present in the suspensions when they are dried, and these may influence the survival of the organisms.

It may well be that freezing is and will continue to be a more efficient method of preserving leptospires than drying, but the latter is undoubtedly more convenient for storage and transport, and attempts to improve its efficiency should be pursued. Of paramount importance, too, is further work to ensure that the physiological characteristics of the organisms are not modified by drying.

\section{REPRINT REQUESTS}

Address reprint requests to: Dr. D. I. Annear, Microbiology Department, Royal Perth Hospital, Box X2213, G.P.O. Perth, Western Australia, 6001.

\section{LITERATURE CITED}

1. Alexander, A. D., E. F. Lessel, L. B. Evans, E. Franck, and S. S. Green. 1972. Preservation of leptospiras by liquid-nitrogen refrigeration. Int. J. Syst. Bacteriol. 22:165-169.

2. Annear, D. I. 1956. Preservation of leptospirae by drying. J. Pathol. Bacteriol. 72:322-323. 
3. Annear, D. I. 1962. Preservation of leptospires by drying from the liquid state. J. Gen. Microbiol. 27:341-343.

4. Annear, D. I. 1962. Recoveries of bacteria after drying on cellulose fibres. Aust. J. Exp. Biol. Med. Sci. 40:1-8.

5. Annear, D. I. 1964. Recoveries of bacteria after drying in glutamate and other substances. Aust. J. Exp. Biol. Med. Sci. 42:717-722.
6. Annear, D. I. 1965. Recoveries of Strigomonas oncopelti, leptospirae and Reiter's treponeme from desiccates after storage for five years. Aust. J. Exp. Biol. Med. Sci. 43:683-684.

7. Cruickshank, R. (ed.). 1965. Medical microbiology. E. \& S. Livingstone, Ltd., Edinburgh.

8. Otsuka, S., and K. Manoko. 1961. Studies on the preservation of leptospirae by freeze-drying. Jap. J. Microbiol. 5: 141-148. 